\title{
Mathematics education in the spotlight: Its purpose and some implications
}

\author{
Jennie Golding* - UCL Institute of Education, UK
}

\begin{abstract}
This article proposes a reframing of the purposes of mathematics education for the twenty-first century that combines apparently divergent philosophical approaches, arguing that the consequent empowerment should as a matter of individual equity be available to all young people (as well as of benefit to wider society). It suggests that the global mathematics attainment 'spotlight', and the English policy context in particular, offer both opportunities and constraints for the development of such a high-quality mathematics education. The article also discusses the challenging implications for the curriculum, and for the nature of teacher expertise, particularly subject-specific expertise, that is needed.
\end{abstract}

Keywords: mathematics education; human flourishing; powerful knowledge; qualification; socialization; subjectification

\section{Introduction}

There is a global focus on mathematics attainment as a gateway to economic progress, with the leaders of many Western nations anxious to see improved results in international performance indicators such as the Programme for International Student Assessment (PISA) or Trends in International Mathematics and Science Study (TIMSS) (Baird et al., 2011). In parallel, there is a widespread refocusing of mathematics curricular which aims to better combine content (facts, procedures), deep conceptual understanding and mathematical processes - and so enable fluency, mathematical reasoning and mathematical problem-solving, thought to be prerequisites for effective mathematical functioning in the twenty-first century. This is hard, and not being achieved at scale (Eurydice, 2011; Spillane, 2004). These twin focuses serve together to create a high-stakes mathematics education environment.

In England, one feature of this has been growing awareness of 'curriculum coherence' (Schmidt and Prawat, 2006): the alignment of curriculum, assessment, teacher development, resources, etc., so that contradictions are not set up and professionals are not subject to contradictory incentives and targets. Here I analyse, in particular, relationships between mathematics-related curriculum intentions (where by 'curriculum' I mean all planned school-related experiences) and the development of appropriate teacher expertise, where at present I restrict the argument to human 'teachers'.

I suggest that the global policy context offers opportunities for a reappraisal of the role of mathematics within the school curriculum, and of the consequent mathematics-specific capacity (Golding, 2017a) needed by teachers of mathematics. Such an evaluation builds on agreed purposes for education. Here I harness Biesta's (2015b) framework to suggest that the apparently divergent approaches adopted by 
Reiss and White (2013) and by Young and Muller (2013) have complementary lenses to offer - but that the implications of their combination for the curriculum, and so for teacher expertise, are profound and demanding. I discuss in particular the systemic and systematic teacher education that is needed to achieve such nuanced purposes. Parts of the article are mathematically technical, but the shape of the argument stands without engagement with those.

\section{What is mathematics?}

The argument depends on some shared conception of mathematics as a discipline. In common with many, but by no means all, mathematicians, I understand the discipline to be concerned with the exploration of, and connections between, patterns that often arise from the external world, their abstraction and their relationships as established through reasoning. Crisan (2017) offers an accessible exegesis of mathematics as a ubiquitous, culturally embedded activity: every human society we know about has developed a mathematical lens on the world and harnessed that lens to aid its flourishing. I develop these ideas further in Golding (2017b) and draw on that here. For an inspirational treatment from a philosophical point of view, I suggest Francis Su's (2017) presidential address to the American Mathematical Society.

Mathematics is therefore part of our cultural heritage, concerned with concepts, but working with culturally developed tools and processes. We are by nature both curious and inventive, and mathematical epistemology features dualities of discovery and creation: its objects include both those embedded in a physical world framed by Tegmark (2008) as completely mathematical in its structure, and discursive constructs with no existence independent of humans, as described by, for example, Adler and Sfard (2017). It is in that sense that mathematical truth is always provisional, openended and relational, situated, social and contextualized.

How then is mathematical knowledge established? Answers to such questions have implications for the way in which mathematics should be learned and taught. Many would argue that what we have in Western society is one, or some, possible mathematical accounts: elsewhere, globally and historically, are further accounts. In Lakatos' (1976) terms, I adopt that fallibilist, rather than absolutist, position. For mathematics to be a shared endeavour, there need to be shared ways of working and of establishing new knowledge in the field, a shared epistemology and syntax. How those are applied when working with novice mathematicians will also be influenced by one's beliefs about how young people (and others) learn.

So what relationship should any mathematics experienced in classrooms have to the mathematics studied in universities or that used outside academia? In Golding (2017b), which is intended to be accessible to the non-specialist, I argue that school mathematics can, and should, be a near-authentic sub-discipline of mathematics - and that, for example, academic mathematics and the mathematics of the mature user of the discipline comprise further, overlapping sub-disciplines. One implication of this view is that, with such an understanding, school students are indeed novice mathematicians and that one aim of school mathematics is to further draw them into a mathematics subculture. For Bernstein (2000) too, the school subject is a recontextualization of the parent discipline, with organizing epistemic coherence stemming from that discipline, even if for a different purpose. But that too begs questions of its operationalization. Note that already, above, we have alternative, or perhaps complementary, lenses on mathematics as discovered or invented. This dualism is reflected in perspectives on the purposes of education as student-centred, coming to understand and harness the 
(natural and social) world, with 'learning' equivalent to 'comprehension', or as worldcentred, disrupting and decentring the student. Both could be argued to support human flourishing. I therefore turn next to a consideration of the purpose of education, and the implications of that for mathematics.

\section{The purpose of education and the role of mathematics within that}

I argue that the purpose of socially sanctioned (and funded) school education is to nurture young people's range of constructive potential and induct them into the culture of their society, so that they mature into fulfilled and well-rounded adults who are able to contribute to and constructively critique society. Education exposes young people to that which is not easily accessible elsewhere, inducting them into social ways of being. In so doing, it supports Reiss and White's (2013) 'aims-based' curriculum which argues for a central curriculum design principle of the achievement of human (individual and societal) flourishing. The notion of human 'flourishing' is not uncontested, but here I adopt a neo-Aristotelian view of eudaimonia consistent with Reiss and White's and for which meaning and purpose are prerequisites. However, such a purpose should also introduce young people, inter alia, to Young and Muller's (2013) 'powerful knowledge' by particular social means. There is in principle no fundamental contradiction here, although I shall show there are tensions inherent in achieving both.

Young and Muller (2013) argue that the school curriculum, whatever else it does, should endow young people with disciplinary 'powerful knowledge' - and that historically, they have been short-changed by the rationing of the school curriculum to that which preserves social hierarchies and limits individual potential. 'Powerful knowledge' here is used as a 'master discourse' (Ball, 2008): an organizing language that offers a framing principle for actions and values, presented as an unexceptionable good and so often serving to preclude dissent. Young and Muller (2013) develop an argument for the inclusion of a range of discipline-embedded knowledge, offering access to aspects of human flourishing. However, they do not offer effective criteria for deciding which disciplinary knowledge is to be considered 'powerful'. For mathematics, I suggest genuine powerful knowledge centres on deep and robust conceptual understanding and familiarity with mathematically valued ways of working, as in the argument below focused on times table knowledge. Here, the 'value' stems from the discipline, as developed for twenty-first-century cultures. To harness those concepts, young people of course also need to develop a repertoire of facts, skills and processes that they can use as tools. At the end of their article Young and Muller, I suggest, overplay their argument and refer to 'powerful knowledge' as not only a necessary, but as a sufficient basis for curriculum design (2013: 247).

I propose that Biesta's (2015b) framework of three domains of educational purpose (and function) allows a reconciliation of Young and Muller's (2013) position with that of Reiss and White (2013). Biesta argues that one of the purposes of education is qualification, understood either in a narrow sense (becoming qualified to perform a certain task or job) or in a much wider sense, such that young people are well prepared to thrive in modern, complex societies. Education is also about socialization, initiating children and young people into existing traditions, cultures, ways of doing and ways of being. That socialization also happens unconsciously through teachers' and students' enculturated ways of being (thus also contributing to the reproduction of material and social inequalities). Any educational activity, further, impacts on the qualities of the person: Biesta frames this as subjectification, as it concerns processes 
of being and becoming a flourishing human subject. Importantly, both Biesta's (2015b) and Young and Muller's (2013) uses of 'flourishing' are consistent with that of Reiss and White (2013). These are three qualitatively different domains with regard to which we need to state and justify what we seek our students to achieve. A 'good' education requires attention to all three; although they are not always easily separable, they are sometimes in tension.

Biesta (2015b) points to common, quite narrow definitions of 'learning' and of the 'effectiveness' of some of the enactments currently adopted, which lead to a distortion of these three core purposes. Within those he argues that, in addition to a technical judgement about the effectiveness of our actions and arrangements, there is a need for judgement (phronesis) about the educational desirability of our actions and arrangements, implicitly, for both individual and society (ibid.: 10). Good teachers therefore not only need appropriate knowledge and skills, and induction into an appropriate professional culture, but also educational virtuosity, that is, embodied educational wisdom: the ability to make wise educational judgements about what is to be done, about what is educationally desirable within that socially sanctioned framework.

So what potential tensions are inherent in an attempt to go beyond a simplistic, and binary, view of students as either subject or object to accommodate support for both 'powerful knowledge' and 'individual and social flourishing'? I have pointed to an aspiration that young people should be inducted both into society and culture (mathematical and wider) to enable their full participation, and be allowed to develop their own meaning-making and values so as to be equipped to constructively critique those cultures. I would want them to develop autonomy as individuals and embryonic mathematicians, as well as agency as citizens and users of mathematics. In curriculum terms, this requires design for learning as well as direct teaching with wisdom that leads into a shared world and shared knowledge. However, the balance needed to achieve this requires the exercise of a deep phronesis - systemically and on the part of individual teachers - that is both sensitive to, and often constrained by, characteristics of the students as individuals and groups, and the prevailing (mathematical and wider) context and culture. In short, there are no easy routes to achieving such aspirations.

In Biesta's (2015b) terms, many English stakeholders are currently perceived to value mathematics education for (narrow) primarily utilitarian qualification purposes. While offering access to techno-scientific literacies and so a gateway to economic prosperity for individuals and society, as mathematics pervades a wide spectrum of our lives, it has the potential to empower much more widely (Smith, 2017). For these ends, but also for wider 'qualification', there is a growing awareness that young people should have a broad mathematics education that equips them less directly, but more potently: skills of mathematical problem posing and solving, and a critical appreciation of the use of mathematical approaches in society (leading to social empowerment through mathematics). Recent curriculum changes in England, as elsewhere, pay at least some nominal attention to this. Such changes support experiencing mathematics not only as discovered and transmitted, but also as invented - although in many cases curriculum coherence still has to be achieved.

I have already pointed to mathematics as part of our cultural heritage; also, given its fundamental role in twenty-first-century economies, it is a matter of both social justice and equity that all young people should be able to access a mathematics education that supports strong mathematical participation and progression. As part of their socialization then, young people should, at an appropriate level, understand the nature of mathematical activity - the syntax and epistemology of the discipline, 
the valued ways of working in the mathematics subculture, their surprises, frustrations and joys - if they are to fully participate in twenty-first-century culture and society. Culturally, I would argue, in common with Ernest (2000: 12):

Learners should gain a qualitative or intuitive understanding of some of the big ideas of mathematics such as pattern, symmetry, structure, proof, paradox, recursion, randomness, chaos, infinity. Mathematics contains many of the deepest, most powerful and exciting ideas created by humankind. These extend our thinking and imaging power, as well as providing the scientific equivalent of poetry, offering noble, aesthetic, and even spiritual experiences.

\section{Implications for curriculum}

What sort of curriculum is implicit in such approaches? Biesta (2015b) points to a current equating of 'learning' with meaning-making that is rather limiting, although Ofsted (2012) shows that in mathematics classrooms in England even this is not being achieved. In the long term, though, students cannot come to use mathematical facts and skills with integrity if they have no known meaning for them. As discussed above, meaning and purpose are necessary, if not sufficient, for human flourishing. So deep conceptual understanding is of value in mathematics education, and overuse of a master discourse such as 'powerful knowledge' can occlude the relative and differential powers of different sorts of knowledge within a discipline.

Further, overemphasis on a reductive 'learnification' (Biesta, 2015b) can compromise the plurality on which a dialogic education supporting socialization, depends. I give two such examples. First, rote knowledge of times tables facts is necessary for efficient mathematical functioning - but times table knowledge is much more powerful if it is recognized as applicable in a range of (external and mathematical) situations, representative of a process that has an associated inverse process, and so on. Secondly, acquaintance with the use of Roman numerals gives (conceptual) access to mathematical aspects of a culture pervasive (among the powerful) in Western civilizations until quite recently, but of greater power is grasping the limitations of that number system in relation to others based on place value, since those offer access to an appreciation of the potency of a concise and precise language for mathematics. There are other routes to such knowledge, but one point for my subsequent argument is that a discipline-informed judgement is needed to discern and harness such distinctions.

I would argue, then, that for Biesta's (2015b) 'subjectification' to be achieved within a mathematics domain, students should come to recognize mathematics both as a tool with which to engage with the world and as a source of personal and social enrichment, cultural heritage and wonder. Further, they need the positive affective resources to harness that education confidently (self-efficacy in relation to an appropriate degree of mathematical functioning, resilience, collaborative and learning dispositions, etc.). It follows that Young and Muller's (2013) 'powerful knowledge' in terms of mathematics, if it is to be more than a slogan, must transcend facts and procedural skills to include knowledge of valued ways of harnessing imagination and creativity to deductive reasoning, pattern-making, analysis and synthesis. It can often spring from the modelling of situations in the external world and might be applied to solve problems in those contexts, but also includes the mathematically internal study of the implications of models and mathematical structures. To the extent that such knowledge is held socially and contextually, the means of accomplishing this need must include the social. 
Young people should therefore not be restricted to learning about concepts and facts, skills and processes, although they need all those. They need also to understand that the discipline of mathematics itself is the authority. The messiness and debate and choices of the discipline are often hidden unnecessarily. Further, it is not just the content of the curriculum that is important: if they are to function confidently and effectively mathematically, young people need to experience, in sustained ways, valued mathematical ways of working - and this is part of Biesta's (2015b) 'subjectification'.

Cuoco et al. (1996) frame such ways of working as 'mathematical habits of mind', saying mathematicians are 'pattern sniffers, experimenters, describers, tinkerers, inventors, visualisers, conjecturers, guessers'. For a classroom, Burton (2004) quotes a poster translating this as 'have imaginative ideas, ask questions, make mistakes and use them to learn new things, are organised and systematic, describe, explain and discuss their work, look for patterns and connections, and keep going when it is difficult'. Details vary across the mathematics literature, but the point is that authentic mathematical activity undertaken by genuine mathematics novices is a far cry from that seen in many English classrooms (Ofsted, 2012), where in too many cases, supported by predictable and routine assessments, the teacher (or the textbook) is seen as the source of mathematical authority and the students' job is to reproduce demonstrated approaches to solving standard exercises.

School mathematics education, then, should aim to support increasing participation in the human endeavour of mathematics in an authentic relationship with the discipline (though necessarily restricted by the institutional constraints of schooling). As such, it has contributions to make - for every young person - to each of Biesta's (2015b) purposes of 'qualification', 'socialization' and 'subjectification'. However, teachers of mathematics also have a moral purpose that is wider than induction into the discipline: they are also the teacher of the whole young person and, for Reiss and White (2013), have responsibility for enabling their wider flourishing, as well as developing their capacity to support the flourishing of others. I shall show below that the implications of this for teacher education are challenging: the teacher too needs to develop appropriate professional qualification, socialization and subjectification.

\section{Expertise for teaching mathematics}

It is an attractive argument that the education of a young person (or, more typically, group of perhaps $3+$ young people) is too important to be left to the discretion of individual teachers. But there is a balance to be found, and Biesta (2015a) convincingly calls for a collective, but non-identical, practice. I suggest that a high-level articulation of shared purposes for education in terms of qualification, socialization and subjectification, and consequent decisions about educational curriculum structures, are the province of government, though drawing substantially on culturally embedded phronesis. Medium-level curriculum decisions should, given the stated purposes of education, lie with education and disciplinary, including pedagogic, community experts. The role of a knowledgeable and (widely) effective professional teacher then is to enact those with phronesis (practical wisdom) in a particular context, for the educational benefit of students, fulfilling the range of purposes of education in a balanced way that will change as time and context change. Such a position is not currently fully supported in English policy, though it is in some other developed jurisdictions. I demonstrate below that large parts of the related detail are dependent on context-specific and professional subject-related knowledge, both of which reside in the expertise of teachers rather than government. 
Addressing this, and complementary to Biesta's (2015b) reclamation of education from 'learnification', Fenstermacher (1986: 39-40) says teaching includes:

instructing the learner on the procedures and demands of the studenting role, selecting the material to be learned, adapting that material so that it is appropriate to the level of the learner, constructing the most appropriate opportunities for the learner to gain access to the content ..., monitoring and appraising the student's progress, and serving the learner as one of the primary sources of knowledge and skill.

Here we come to one of the biggest challenges associated with trying to establish principled approaches to mathematics education. Teaching in order to build up powerful knowledge, given the range of these roles and more, is hard. It is clear that enacting much of the above requires discipline-specific knowledge. Minimally, this includes knowledge of the culture-specific school sub-discipline at an appropriate level; of the disciplinary language, organization, approaches and ways of establishing knowledge; its epistemology and syntax; shared values and ways of working. Teachers have to understand its recontextualization for the classroom and the ways of re-presenting mathematics so as to make it accessible to young people. They have to know the ways in which students interact with mathematics, the potential barriers to that and the systemic disciplinary expectations as communicated in curriculum documents and assessments. Teachers' knowing, of doing and of being all have discipline-specific elements if they are to make the potential of this school mathematics available for learning. As described above, that is not the sum of the demands on teachers of mathematics, of course, for they are not just teachers of mathematics, but of whole young people. Each of these roles entails judgement (phronesis) in its enactment: good teaching is inherently situated and context-dependent.

So what subject-specific knowledge do teachers of mathematics need? In a mathematics classroom, authority derives from at least the parent discipline, knowledge of the subject for teaching and the teacher's pedagogical knowledge, including their subject-specific pedagogical knowledge. That knowledge, held at a deep level, is needed for 'curriculum-making' (Lambert and Biddulph, 2015), that is, the transformation and synthesis of the written intended curriculum into a coherent and meaningful classroom-enacted whole in ways that are consistent with the values of the professional community and its standards of integrity and wise judgement.

What is the nature of the requisite discipline-specific knowledge? Ball et al. (2008) show that teacher effectiveness appears to have no simple relationship with, for example, the number of discipline-related college courses they have taken, although there is clearly a need for a confidence and deep grasp of mathematics beyond the level they currently teach at. Ma (1999), though, demonstrates the need for teachers to have a 'profound understanding of elementary mathematics'. It is knowledge which differs from that of a numerate layman, or professional user of the discipline, but also differs profoundly from that of an academic mathematician. Further, we have seen above that it includes a discipline-specific appreciation of the purpose and role of mathematics education in contributing to the qualification, socialization and subjectification of the student.

For example, a primary schoolteacher whose children have a reasonable grasp of whole numbers then has to teach that three-quarters is part of a whole (and which whole). It is also three lots of a quarter of one or more identical wholes, three identical wholes shared equally among four, an operator (as in three-quarters of a pile of Smarties), a number in its own right, with its own position on a number line, a ratio, 
an equivalence class of fractions, and so on. All these concepts have to be planned for, introduced and worked with at appropriate times, with a range of appropriate representations, in culturally and contextually appropriate ways, and young people supported in making connections between these different conceptualizations, until, in the long term, they compress that understanding into a single, but multifaceted, idea of 'three-quarters' that they can use as a tool within and beyond mathematics. (For the academic mathematician, in contrast, 'three-quarters' is one of the 'rationals' defined from whole numbers in an abstract, algebraic way: the related two sub-disciplines require different knowledge.) Further, the same primary schoolteacher in the melee of the classroom moment, has to comprehend, evaluate and decide how to deal with pupils' alternative understandings as they develop, responding in ways that build not only that student but others' mathematical ways of participating meaningfully in society, their socialization into mathematics and wider cultures, and the wider subjectification of the student. That is no small task, even for a mathematics specialist, but primary schoolteachers in England typically teach across the curriculum: the consequent holistic knowledge of each young person is highly valued culturally (Alexander, 2010), but it might also be argued to contribute to that subjectification.

Teaching for mathematics at any level therefore requires a great breadth and depth of subject-related knowledge - and much more - if it is to be effective. One widely used theorization of necessary mathematics-specific knowledge is Ball et al.'s (2008) 'egg', which builds on Shulman's (1987) generic typology of teacher knowledge. This divides the subject-specific knowledge needed into subject knowledge and subject pedagogic knowledge. The former encompasses not only what the mathematically competent adult commonly needs, but also deep knowledge of its different conceptualizations, where those might be encountered and the relationship between them (as in the example of 'knowing' three-quarters above) as well as the links between each idea to others within or beyond mathematics. Pedagogically, the teacher also needs to know, inter alia, where those different conceptualizations sit within the curriculum; the progressions to and from those (as structured by the discipline); how concepts might meaningfully and constructively be re-presented to students and in what contexts; how students, and particularly the students in this class, might typically understand them in 'different' ways, as well as students' affective needs in relation to mathematics; how to elicit related developing understanding and harness that constructively, and how to support a grasp of the related mathematically valued connections both within and beyond mathematics.

An alternative, higher-level theorization analysing disciplinary knowledge that is exposed in classrooms comes from Rowland et al. (2005). From classroom observations, they show that in teaching mathematics, teachers draw on mathematical 'Foundation' knowledge of mathematics, students in relation to mathematics, curriculum, etc.; on 'Transformation' knowledge which is the transformation of mathematics into a form that allows students to access its underlying motivations, structures and warrants; on 'Connection' knowledge within and beyond mathematics, of the authentic use of mathematical thinking and of utilitarian applications; and on 'Contingency' knowledge of how to field both questions and a variety of responses from students, some of which will be based on challenging and non-standard conceptualizations of emerging ideas - and in mathematically constructive ways. Again, what is seen to be necessary is a rich and deeply connected discipline-embedded network that can be drawn on with phronesis to further the range of purposes of education. Although in both cases the categories have 'fuzzy' boundaries when operationalized, they serve to point us to complex aspects of teachers' subject-specific knowledge that are critical to good 
teaching as well as being highly situated and contextualized. These conceptualizations therefore have implications for both initial teacher training and teachers' continuing development, but they only acquire their full meaning and import when they are applied in relation to the underlying valued purposes of education.

\section{The development of mathematics teacher expertise}

In Golding (2015), I argue that such expertise needs specialized development, including in initial and early career teacher education. Teacherly knowledge has a dual nature, comprising both theoretical and practical aspects. Not only do teachers of mathematics have to understand, for example, the multifaceted nature of threequarters, but they have to be able to operationalize education for a specific group of children who bring particular mathematical and whole selves to the classroom, and who will respond differentially to particular personal and mathematical approaches, and mathematical explanations and embodiments. Education for such complexity requires expertise in teacher development in each of the theoretical (mathematics education, teacher education, child psychology, etc.) and classroom domains - and the development of practical wisdom. Schools do not typically house experts in teacher theoretical education, but they do house experts in classroom teaching for their own context. Very often, then, effective initial teacher education can be achieved through strong partnerships between university education departments and schools, and models of teacherly phronesis might be found in either. The former, typically staffed by experienced teachers further resourced to develop expertise in teacher education, can also provide time and knowledgeable support for developing deep, informed reflection, building of goal-related dispositions and knowledge of the evidence base and the opening up of possibilities that are often not available in schools, which, by their nature, are focused on the education of young people. From the earlier discussion, it is clear that for all teachers of mathematics, substantial parts of this early development need to be subject-specific.

The discourse surrounding further teacher development in recent years, and globally, has been one of 'teacher deficit'. There is substantial evidence that in schools in England at present there is indeed a lack of appropriate mathematics knowledge for teaching that significantly limits the quality of mathematics learning available (e.g. Ofsted, 2012), however worthy the intentions of the curriculum. However, there is some evidence that, given appropriate subject-specific and generic foundations (the precise nature and extent of which are yet to be established), together with sufficient time, goal-related dispositions and appropriate access to expertise (some of which might be peer-collaborative), experienced teachers are often able to develop in new directions and depths in semi-autonomous ways (e.g. Chan et al., 2018; Golding et al., 2018).

Yet none of this is sufficient to ensure the development of teachers' practical wisdom or phronesis, which needs all of the above and more. As Biesta (2015a) argues, competences are necessary, but not sufficient, for good teaching, which depends on the effective harnessing of those competences for agreed valued domains of educational purposes. I argue, consistent with Biesta (2015a), that true phronesis can only be developed with experience, together with professional example and sustained, informed reflection. Again, there is a clear but different role apparent for early contextualized experiences in school settings supported by deep reflection at the university. Both of these, ideally, would be underpinned by experienced, effective teachers able to model the exercise of professional phronesis. Where a system has, for 
whatever reason, limited capacity to model the range of such 'professional virtue', the challenges of developing effective (mathematics) education are of course exacerbated.

\section{How does the policy context in England impinge on possibilities for mathematics education?}

In a high-stakes policy environment, it is difficult for policymakers, end-users and those involved in mathematics education alike to step back and reflect in depth about what it is we are trying to achieve in mathematics education. It is not easy to expose the variety of aims and purposes assumed by different policy 'players' (Ball et al., 2011), so that those are held up to scrutiny and the possibilities for workable, shared values debated. I would argue that reasonable performance on widely valued international attainment measures should be expected from a wealthy nation, but questions should be asked about whether such measures together reflect the range of socially valued mathematics education (and other) outcomes. TIMSS measures performance on curriculum-close mathematics questions, whereas PISA assesses the solving of rather more contextualized tasks. Yet political responses rarely seem to be based on the nature of what is measured (Baird et al., 2011). I would suggest that even good performance on both of these, which taken together arguably support mathematically 'powerful knowledge', is not sufficient for either personal or societal flourishing.

Further, in England, teachers of mathematics, from Early Years upwards, are dealing with rapid and frequent curriculum changes. They are also working in highstakes assessment environments whose focus, for mathematics at least, has recently been broadened but which still, by its nature, privileges short-term performance. One argument in favour of the high value given to academic disciplinary functioning (Gibb, 2017) is that it enables all young people to access the knowledge that, in a more liberal education environment, would be available only to the privileged. But a consequence of rapid and aspirational change is that teachers have very little time or energy available for deep reflection on, or considered preparation or development of, their teaching. It is not yet obvious that the combination is of net benefit to young people - though recent changes have yet to bed down.

It is clear, then, that if we want to achieve an authentically mathematically well-equipped population, so that both individuals and society flourish, we certainly need the supply and development of sufficient mathematically knowledgeable and pedagogically effective teachers, able to develop an enacted curriculum in disciplineknowledgeable ways and exert professional judgement that builds up a balanced (wider) qualification, socialization and subjectification for their context. There are both opportunities and constraints associated with this.

Mathematics education is currently valued by a range of stakeholders, including policymakers. There is therefore a (relatively) good level of financial investment available, and an in-principle will to develop teacher education in ways that support that. There is also an opportunity to move away from a 'deficit' model of teachers' continuing education and build on the undoubted potential of well-prepared teachers to drive their own (both subject-specific and generic) development, learning from their teaching (e.g. Chan et al., 2018) and from opportunities to work collaboratively with expert others, supported by bespoke time and moderate funding (e.g. Golding et al., 2018).

On the other hand, while a high-stakes accountability regime and valuing of international performance measures of mathematical functioning and more can support mathematical education development, accompanying national assessments 
that do not fully support curriculum intentions serve to undermine any such broad and balanced curriculum provision (Neumann et al., 2016). The long-standing lack of subject-specific expertise among teachers (Ofsted, 2012), coupled with recruitment and retention challenges (National Audit Office, 2016) can threaten the contextual, reflexive, profoundly phronesis-dependent and iterative elements of the work of good teaching.

In parallel, English teacher education, both initial and continuing, is currently in a state of flux, with fragmented and increasingly generic provision, often led by schools and sometimes with no input from higher education. So, despite a range of threats to the health of a subject perceived to be important, the development of mathematics knowledge for teaching is being marginalized. The arguments above make it clear that such a situation undermines both the attainment of an effective mathematics education and the viability of a balanced, phronesis-rich education that is balanced across purposes.

Moving to a point where we have a consensus about the needs of initial and continuing teacher development, though, assumes attainment of a shared view of the kind of teaching that is needed in schools. Those sustained conversations between professionals, policymakers and stakeholders about what we value in education, why and how that might be achieved are important. It would be naive to suggest that previous teacher education systems were ideal: they operated in a national context with less competition for mathematics expertise and with fewer pressures of performativity on teacher educators.

We now know, though, far more than we used to about how young people learn mathematics, and what teachers need to be able to support that. We need to capitalize on that knowledge in a coherent and long-term way and to recognize the need for a theorization of discipline-specific expertise in both subject and subject pedagogical arenas. And because in England we patently do not yet have a shared vision for education, let alone mathematics education, the present challenges offer every incentive to develop those in the interests of achieving individuals and societies that are genuinely flourishing.

Emerging English - and global - mathematics curricula for students aged 5 to 18 do, to a large extent, embody widely held values of mathematics education communities. As we acquire evidence of what is working well and why, we should find ways to support the evolution of those curricula and the surrounding system (assessment, CPD, resources, etc.) into a coherent whole, which values education for individual and societal flourishing without sacrificing the sorts of knowledge that keep open the doors to social equity. A variety of initial teacher education models and moves to mixed models of teacher development challenge our assumptions about how teachers learn, when and how. That is healthy - provided it is also regularly and effectively evaluated, and changes are made in response to that evaluation, so that we do not persist in perpetuating mediocrity wherever it occurs.

\section{Conclusions}

This article suggests that the global mathematics attainment spotlight offers both opportunities and constraints for the development of high-quality mathematics education - and for the debate about what that comprises. I use Biesta's (2015b) framework for the purposes of education to argue that mathematics has a role to play in contributing to both Reiss and White's (2013) 'education for human flourishing' and Young and Muller's (2013) 'powerful knowledge', although achieving a wise balance is 
demanding, not only for policymakers but also for individual teachers. For mathematics, though, I critique Young and Muller's use of the term 'powerful knowledge', which minimally takes the form of deep conceptual understanding. For any one concept, this is complemented by a range of known facts and established skills that enable its effective harnessing for a variety of socially valued and individually enriching purposes. However, it remains the case that different mathematics conceptual areas remain differentially 'powerful'. The details of the appropriate range and scope of conceptual access should remain contextually and socially determined, with due regard for the global nature of the economy and society in which twenty-first-century young people will live. The consequent mathematical - and wider - empowerment should be available to all young people as a matter of equity and social justice (as well as benefit to wider society).

Such a mathematics education, though, only represents part of the responsibility of the teacher of mathematics, who should also look to the development and flourishing of the whole young person, exercised with appropriate phronesis. I discuss the consequent nature of mathematics teacher expertise, and particularly subjectspecific expertise, that is needed to facilitate such education, arguing that the initial development of that expertise to a well-evidenced threshold level should be supported by centres of teacher education expertise which, because of the dual theoretical and practical nature of that education, will often comprise partnerships of universities with schools and colleges. Beyond that initial threshold, I argue that teachers are often best served by the opportunity to build on that initial expertise, with sufficient space and time for deep and collegially informed reflection, supported as appropriate by peer or external expertise, and so moving away from a model of a deficit in teacher capacity. I suggest the current 'high stakes' policy context in England and elsewhere offers an opportunity to prioritize a theorization of subject-specific teacher expertise that would be situated in the professional rather than the policy domain, and to fund both a focused recapacitation of the mathematics education teaching force and a movement towards such a professional model of further learning.

\section{Notes on the contributor}

Jennie Golding worked in school-based mathematics teaching and teacher education in England and internationally before moving to higher education. She is actively involved in influencing a range of education policy development in evidence-informed ways. Her research centres around the classroom enactment of mathematics education policy for students aged 5 to 18 and to the related teacher development.

\section{References}

Adler, J. and Sfard, A. (eds) (2017) Research for Educational Change: Transforming researchers' insights into improvement in mathematics teaching and learning. London: Routledge.

Alexander, R. (ed.) (2010) Children, Their World, Their Education: Final report and recommendations of the Cambridge Primary Review. London: Routledge.

Baird, J.-A., Isaacs, T., Johnson, S., Stobart, G., Yu, G., Sprague, T. and Daugherty, R. (2011) Policy Effects of PISA. Oxford: Oxford University Centre for Educational Assessment.

Ball, D.L., Thames, M.H. and Phelps, G. (2008) 'Content knowledge for teaching: What makes it special?'. Journal of Teacher Education, 59 (5), 389-407.

Ball, S.J. (2008) 'New philanthropy, new networks and new governance in education'. Political Studies, 56 (4), 747-65. 
Ball, S.J., Maguire, M., Braun, A. and Hoskins, K. (2011) 'Policy subjects and policy actors in schools: Some necessary but insufficient analyses'. Discourse: Studies in the Cultural Politics of Education, $32(4), 611-24$.

Bernstein, B. (2000) Pedagogy, Symbolic Control and Identity: Theory, research, critique. Rev. ed. Lanham, MD: Rowman and Littlefield.

Biesta, G. (2015a) 'How does a competent teacher become a good teacher? On judgement, wisdom and virtuosity in teaching and teacher education'. In Heilbronn, R. and Foreman-Peck, L. (eds) Philosophical Perspectives on Teacher Education. Chichester: Wiley-Blackwell, 3-22.

Biesta, G. (2015b) 'What is education for? On good education, teacher judgement, and educational professionalism'. European Journal of Education, 50 (1), 75-87.

Burton, L. (2004) '"Confidence is everything" - perspectives of teachers and students on learning mathematics'. Journal of Mathematics Teacher Education, 7 (4), 357-81.

Chan, M.C.E., Clarke, D.J., Clarke, D.M., Roche, A., Cao, Y. and Peter-Koop, A. (2018) 'Learning from lessons: Studying the structure and construction of mathematics teacher knowledge in Australia, China and Germany'. Mathematics Education Research Journal, 30 (1), 89-102.

Crisan, C. (2017) 'Mathematics'. In Standish, A. and Sehgal Cuthbert, A. (eds) What Should Schools Teach? Disciplines, subjects and the pursuit of truth. London: UCL Institute of Education Press, 20-37.

Cuoco, A., Goldenberg, E.P. and Mark, J. (1996) 'Habits of mind: An organizing principle for mathematics curricula'. Journal of Mathematical Behavior, 15 (4), 375-402.

Ernest, P. (2000) 'Why teach mathematics?'. In Bramall, S. and White, J. (eds) Why Learn Maths? London: Institute of Education, 1-14.

Eurydice (2011) Mathematics Education in Europe: Common challenges and national policies. Brussels: Education, Audiovisual and Culture Executive Agency.

Fenstermacher, G.D. (1986) 'Philosophy of research on teaching: Three aspects'. In Wittrock, M.C. (ed.) Handbook of Research on Teaching. 3rd ed. New York: Macmillan, 37-49.

Gibb, N. (2017) 'Speech: Nick Gibb: The importance of an evidence-informed profession: School Standards Minister Nick Gibb addresses Buckingham University PGCE students'. Online. www. gov.uk/government/speeches/nick-gibb-the-importance-of-an-evidence-informed-profession (accessed 18 July 2018).

Golding, J. (2015) 'What has the Coalition Government done for the development of initial teacher education?'. London Review of Education, 13 (2), 113-24.

Golding, J. (2017a) 'Mathematics teachers' capacity for change'. Oxford Review of Education, 43 (4), 502-17.

Golding, J. (2017b) 'Is it mathematics or is it school mathematics? Presidential address to the Mathematical Association, April 2017'. Mathematical Gazette, 101 (552), 385-400.

Golding, J., Smith, C. and Blaylock, M.J. (2018) 'Learning to teach contextualized problem-solving in a non-calculus mathematics pathway'. Teaching Mathematics and Its Applications, 37 (2), 69-83.

Lakatos, I. (1976) Proofs and Refutations: The logic of mathematical discovery. Cambridge: Cambridge University Press.

Lambert, D. and Biddulph, M. (2015) 'The dialogic space offered by curriculum-making in the process of learning to teach, and the creation of a progressive knowledge-led curriculum'. AsiaPacific Journal of Teacher Education, 43 (3), 210-24.

Ma, L. (1999) Knowing and Teaching Elementary Mathematics: Teachers' understanding of fundamental mathematics in China and the United States. Mahwah, NJ: Lawrence Erlbaum Associates.

National Audit Office (2016) Training New Teachers. London: National Audit Office.

Neumann, E., Towers, E., Gewirtz, S. and Maguire, M. (2016) A Curriculum for All? The effects of recent Key Stage 4 curriculum, assessment and accountability reforms on English secondary education. London: National Union of Teachers.

Ofsted (2012) Mathematics Made to Measure. London: Ofsted.

Reiss, M.J. and White, J. (2013) An Aims-Based Curriculum: The significance of human flourishing for schools. London: Institute of Education Press.

Rowland, T., Huckstep, P. and Thwaites, A. (2005) 'Elementary teachers' mathematics subject knowledge: The knowledge quartet and the case of Naomi'. Journal of Mathematics Teacher Education, 8 (3), 255-81.

Schmidt, W.H. and Prawat, R.S. (2006) 'Curriculum coherence and national control of education: Issue or non-issue?'. Journal of Curriculum Studies, 38 (6), 641-58.

Shulman, L.S. (1987) 'Knowledge and teaching: Foundations of the new reform'. Harvard Educational Review, 57 (1), 1-22. 
Smith, A. (2017) Review of Post-16 Mathematics in England. London: Department for Education. Spillane, J.P. (2004) Standards Deviation: How schools misunderstand education policy. Cambridge, MA: Harvard University Press.

Su, F. (2017) 'Mathematics for human flourishing'. The Mathematical Yawp blog, 8 January. Online. https://mathyawp.wordpress.com/2017/01/08/mathematics-for-human-flourishing/ (accessed 18 July 2018).

Tegmark, M. (2008) 'The mathematical universe'. Foundations of Physics, 38 (2), 101-50.

Young, M. and Muller, J. (2013) 'On the powers of powerful knowledge'. Review of Education, 1 (3), 229-50. 